\title{
Signal to noise ratio of layer-oriented measurements for multiconjugate adaptive optics ${ }^{\star}$
}

\author{
D. Bello ${ }^{1,2}$, J.-M. Conan ${ }^{2}$, G. Rousset ${ }^{2}$, and R. Ragazzoni ${ }^{3}$ \\ ${ }^{1}$ GRANTECAN, S.A. C/Vía Láctea, s/n, CP 38200, La Laguna, Spain \\ 2 ONERA, DOTA, BP 72-29, Av. Division Leclerc, 92322 Châtillon Cedex, France \\ 3 OAA, Largo E. Fermi 5, 50125 Firenze, Italy
}

Received 16 April 2003 / Accepted 8 August 2003

\begin{abstract}
Multiconjugate adaptive optics (MCAO) employing several deformable mirrors conjugated to different altitudes has been proposed in order to extend the size of the corrected field of view. A three dimensional measurement of the turbulent volume is needed in order to collect the information to control the deformable mirrors. Given a set of guide stars in the field of view, this can be done either using star-oriented or layer-oriented techniques. In the star-oriented measurement each wavefront sensor is coupled to a guide star while in layer-oriented techniques, wavefront sensors are coupled to different layers in the atmosphere and each of them collect light from the whole set of guide stars. This type of measurement is more exactly called optical layer-oriented (OPTLO) as the co-addition of light is done optically. The same information can also be obtained by combining, in a numerical way, star-oriented measurements. This hybrid approach is called numerical layeroriented (NUMLO). In order to compare their performance, we present an analytical study of the signal to noise ratio (SNR) in the optical and numerical layer-oriented measurements. Optical layer oriented measurements are shown to be more efficient in the regime of faint flux and a large number of guide stars, while low detector noise allows numerical layer-oriented schemes to be more efficient in terms of $S N R$.
\end{abstract}

Key words. instrumentation: adaptive optics - techniques: high angular resolution - methods: analytical - atmospheric effects - telescopes

\section{Introduction}

The image quality of a ground-based telescope is severely degraded due to the atmospheric turbulence. Fluctuations of the air refraction index are responsible for the loss of resolving power for ground-based telescopes. This resolving power no longer depends on the telescope diameter, but on the Fried parameter, which at the best observatories rarely exceeds $50 \mathrm{~cm}$ in the visible range. In order to overcome this limitation, the use of an active optical element to correct the wavefront distortions in real time was proposed by Babcock (1953). This technique is called adaptive optics and 35 years after being proposed, once the technical solutions were found for a large number of problems, COME-ON became the first adaptive optics system devoted to astronomy (Rousset et al. 1990). Adaptive optics systems are now working on a large number of telescopes (see for instance Rousset et al. 2000; Wizinowich et al. 2000; Graves et al. 2000; Rousset et al. 2002) allowing diffraction-limited

Send offprint requests to: D. Bello, e-mail: cbello@ll.iac.es

* Appendix A is only available in electronic form at http://www.edpsciences.org images to be obtained. However, due to the vertical distribution of the atmospheric turbulence, the size of the corrected field of view (FOV) is limited to a few arcsecs. In order to extend the compensated FOV, the concept of multiconjugate adaptive optics (MCAO) has been proposed (Beckers 1989; Dicke 1975) in which several deformable mirrors are conjugated to different altitudes. These deformable mirrors are controlled using the information about the phase measured using several guide stars. A star-oriented approach was first proposed (Tallon \& Foy 1990) in which a wavefront sensor is coupled to each guide star and then all the information is processed and employed to control each deformable mirror (see Fig. 1a). More recently another concept, called the layer-oriented approach, has been proposed by Ragazzoni et al. (2000), Ragazzoni (2000) (see Fig. 1b). It is based on measuring the phase with a single wavefront sensor with one or more sensing planes which can be optically conjugated to given layers in the atmosphere. A deformable mirror (DM) is associated with each of the wavefrontsensing planes, and the wavefront sensor collects light from the whole set of guide stars. This technique was first conceived with the idea of making this kind of measurement in an optical 


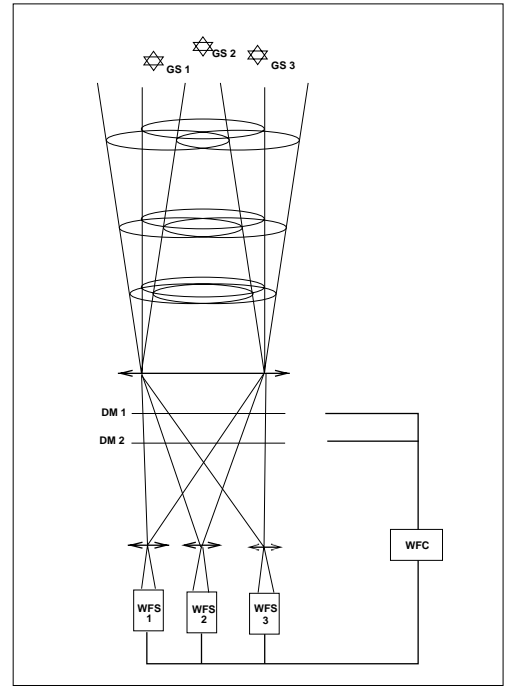

(a) Star-oriented approach.

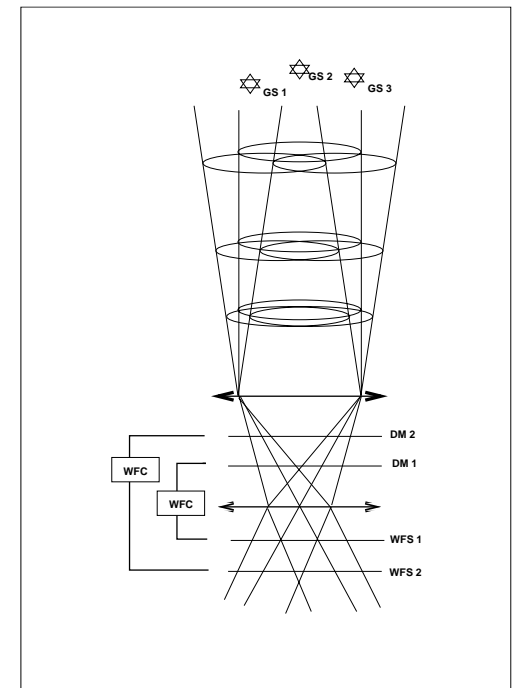

(b) layer-oriented approach.

Fig. 1. Schemes of star-oriented and layer oriented approaches. GS = guide star, WFS = wavefront sensor, WFC = wavefront controller and $\mathrm{DM}=$ deformable mirror.

way. However, measurements of the same nature can be obtained by numerically combining star-oriented measurements where each wavefront sensor is coupled to a single guide star.

The objective of this work is to compare star-oriented and layer-oriented measurements in terms of signal to noise ratio $(S N R)$. But in order to compare $S N R$ values, measurements of the same nature must be used. This is why we chose to translate the problem to comparing numerical layer-oriented and optical layer-oriented measurements.

Section 2 explains in detail the differences between optical and numerical layer-oriented measurements. Analytical formulae for the $S N R$ corresponding to each technique are developed in Sect. 3, and a discussion is given in Sect. 4. An Appendix is included (Appendix A) which is devoted to the comparison of the $S N R$ of the two techniques for several study cases.

\section{Optical and numerical layer-oriented measurements}

Figure 1 shows the schemes for the star-oriented and layeroriented approach. In the star-oriented approach, each wavefront sensor is coupled to a single guide star. The information from all the wavefront sensors is combined and processed in the wavefront controller and allows one to obtain the commands for the deformable mirrors of the system. We consider wavefront sensors of the Shack-Hartmann type (Rousset 1999) or pyramid (Ragazzoni 1996), measuring the gradient of the wavefront. Each wavefront sensor collects information from the column of turbulence along the direction of the guide star it is coupled to. If we consider an atmospheric turbulence with $L$ layers:

$\frac{\partial \Phi^{s}(\boldsymbol{x})}{\partial x}=\frac{\partial}{\partial x} \sum_{l=1}^{L} \phi_{l}\left(\boldsymbol{x}+h_{l} \theta_{\mathbf{s}}\right)$

where $\phi_{l}$ is the phase error introduced by the $l$ th layer, and $\Phi^{s}$ is the wavefront measured using the sth guide star. The same applies to the phase derivative along direction $y$. Point $\boldsymbol{x}$ in the pupil plane corresponds to point $\boldsymbol{x}+h_{l} \theta_{\mathbf{s}}$ in layer $l$, at altitude $h_{l}$, where $\theta_{\mathrm{s}}$ is a vector parallel to the plane of the aperture which gives the position of the guide star. The sampling of the volume of turbulence using several analysis directions allows one to reconstruct the turbulent volume. Figure 2 a shows a block diagram of star-oriented measurements when using three guide stars. We will assume in this development that the near-field approximation applies and that the wavefronts are geometrically propagated through the atmosphere.

In the optical layer-oriented approach (OPTLO), a single wavefront sensor collects light from the ensemble of guide stars (Ragazzoni et al. 2000). For instance, pyramids can be placed at the focal plane, and a common objective allows pupil images from different guide stars to be superimposed. A beam splitter is employed to split light between the different wavefrontsensing planes which are conjugated to different specific altitudes in the turbulent profile. Each of these wavefront-sensing planes will see the layer it is conjugated to, but also superimposed wavefronts corresponding to the other layers. When the loop is closed, each detector senses the perturbation that occurred in its own layer, while the perturbations of the other layers are cancelled by the correction provided by the other DMs. This approach allows the system to have independent "classical adaptive optics" loops, linking each deformable mirror with its associated wavefront-sensing plane (see Fig. 1b). The measurement done for each detector plane can be written as a linear combination of the star-oriented measurements weighted by the intensity of each of the guide stars. A wavefront-sensing plane $d$ conjugated to a turbulent layer at altitude $h_{d}$ will measure, in the ideal case where there is no noise:

$\frac{\partial \Phi(\boldsymbol{x})}{\partial x}=\frac{\sum_{s=1}^{N_{\mathrm{gs}}} I_{s} \times \sum_{l=1}^{L} \frac{\partial}{\partial x} \phi_{l}\left(\boldsymbol{x}+\left(h_{l}-h_{d}\right) \theta_{\mathbf{s}}\right)}{\sum_{s=1}^{N_{\mathrm{gs}}} I_{s}}$

where $N_{\mathrm{gs}}$ is the number of guide stars, and $I_{s}$ is the intensity of $s$ th guide star (Diolaiti et al. 2001). 


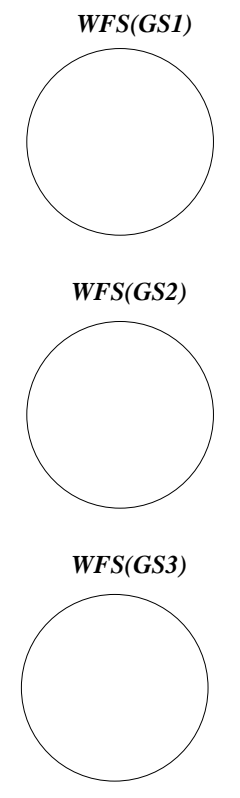

(a) The star-oriented approach.

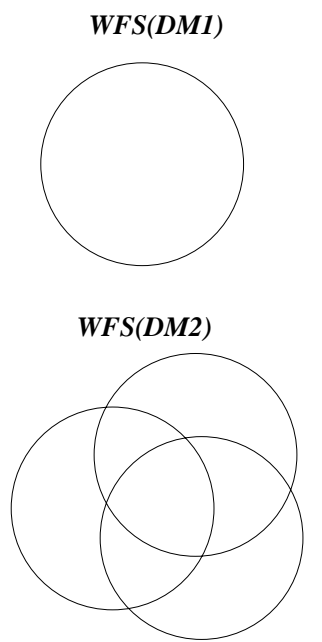

(b) The layer-oriented approach.

Fig. 2. Wavefront sensor measurements in star-oriented and layer-oriented approaches when employing three guide stars and two wavefront sensor planes in the layer-oriented approach, conjugated to DM1 and DM2. For the sake of simplicity, DM1 is conjugated to the pupil plane, and thus in its associated wavefront sensor plane, the footprints of the three guide stars perfectly overlap. This is not the case with DM2, conjugated to a turbulent layer in altitude, where the star footprints are displaced by an amount depending on the star position in the field and on the altitude of the layer.

When a wavefront-sensing plane is placed at the pupil plane, the footprints of each of the stars overlap at the same position (i.e. the telescope pupil) while for the wavefront-sensing planes situated at any other altitude, the measured phase will be the overlap of the phases sampled by each star with the corresponding displacement given by $\boldsymbol{r}=h \theta_{\mathrm{s}}$, where $h=h_{l}-h_{d}$ is the difference between the layer altitude and the wavefront sensing-plane altitude of conjugation (see Figs. $1 b$ and $2 b$ ). In the illustrative case shown in Fig. 2, and assuming that the guide stars all have the same magnitude and that the same spatial and temporal sampling is used in both star-oriented and layer-oriented measurements, the sum of the three staroriented measurements divided by three (the number of stars in this case) would give the layer-oriented measurements in the pupil conjugate sensing plane. When reconstructing the layeroriented measurements in the sensing plane conjugated to a layer at an altitude $h$ in the atmosphere, the star-oriented measurements have to be displaced at the position given by the footprint of the star at that layer before they are summed. When overlap exists, the phase measurement at those points has to be divided by the number of guide stars that contribute to the measurement at those points. When guide stars of different magnitudes are used, the phase measurements are weighted by the intensity of the guide star relative to the total intensity.

Due to the nature of OPTLO measurements where in a closed loop each detector senses to the first order the wavefront perturbations occurring in its conjugated layer, the spatial and temporal sampling ( $d_{l}$ subaperture size, $\Delta t_{l}$ integration time) can be adjusted to the characteristics of layer to which the detector is conjugated (Ragazzoni et al. 2000). The vertical distribution of the $C_{n}^{2}$ profile (Le Louarn et al. 2000) is such that most of the turbulence is frequently in the layers near the ground.
Thus the upper layers will have a larger Fried parameter, and thus the subaperture size in a wavefront-sensing plane conjugated to a high altitude layer can be larger than those employed in a wavefront-sensing plane conjugate to the pupil plane. In the case of a wavefront sensor coupled to a guide star, the spatial sampling is given by the global Fried parameter which is always smaller than the Fried parameter corresponding to each layer.

As we mentioned before, the optical layer-oriented approach is a linear combination of star-oriented measurements (apart from noise considerations) that is done optically. This combination of star-oriented measurements could be also done numerically after the detection of the phase error by each of the wavefront sensors. In this hybrid technique, which is called a numerical layer-oriented scheme (NUMLO), we pass from having a set of measurements per guide star to having a set of measurements per layer. In the star-oriented measurements, the spatial and temporal sampling is matched to the characteristics of the global atmosphere. In order to reproduce measurements of the same nature as OPTLO measurements, the same integration time and integration area must be used. This is done numerically from star-oriented measurements. For instance, if the coherence time of the upper layer is twice the global one, the integration time of the optical layer-oriented measurements in this layer will be twice the integration time employed in the star-oriented measurements and we will have to add two standard frames in order to reconstruct a single frame obtained by means of optical layer-oriented measurements. The same applies to the subaperture size.

In an ideal case, without any noise source, numerical and optical layer oriented measurements would be the same. In fact, the only difference between the measurements done by the two 
Table 1. List of parameters necessary to compute the SNR.

\begin{tabular}{|c|c|}
\hline$N_{\mathrm{ph}}^{l}(r)$ & $\begin{array}{l}\text { Number of photons per second per square meter per bandwidth in a given layer } l \text { of the } \\
\text { atmosphere, depending on the guide stars geometry, and magnitude }\end{array}$ \\
\hline$N_{\mathrm{gs}}^{l}(r)$ & number of stars whose footprints contribute at $r$ in the layer $l$. \\
\hline$\tau$ & Coherence time of the whole atmosphere \\
\hline$\tau_{l}$ & Coherence time of layer $l$ \\
\hline$\Delta t$ & Integration time employed for the whole atmosphere \\
\hline$\Delta t_{l}$ & Integration time employed for layer $l$ \\
\hline$d$ & Sampling pitch for the wavefront measurements in NUMLO approach. \\
\hline$d_{l}$ & Sampling pitch for the wavefront measurements in OPTLO approach, layer $l$. \\
\hline$r_{0}$ & Fried parameter describing the whole atmosphere. \\
\hline$r_{0, l}$ & Fried parameter associated to layer $l$. \\
\hline$r_{0}\left(\lambda_{\mathrm{wfs}}\right)$ & Fried parameter describing the whole atmosphere at the wavefront-sensing wavelength. \\
\hline RON & read-out noise of the $\mathrm{CCD}$ detector. \\
\hline$\alpha_{l}$ & $\begin{array}{l}\text { fraction of light arriving to the detector } l \text { in the optical layer } \\
\text { oriented measurements due to the beam splitter. }\end{array}$ \\
\hline$N_{\text {bg }}$ & number of background photons $\mathrm{s}^{-1} \mathrm{~m}^{-2} \operatorname{arcsec}^{-2}$ \\
\hline$Q_{\mathrm{e}}$ & quantum efficiency (it includes also optical transmission) \\
\hline
\end{tabular}

techniques is due to the effect of noise. Three sources of noise have to be considered: the photon noise linked to the finite star photon flux, the background noise and the detector read-out noise. One can foresee that since the NUMLO measurements have been created from star-oriented measurements where one detector is employed per guide star, the read-out noise increases when the number of guide stars is increased. On the other hand, the photon flux is not divided between different altitude planes in this technique, and thus the number of DMs can be easily increased without degrading the SNR per layer. In the OPTLO a beam splitter is employed and there is a loss of flux in the measurements per layer. This effect will greatly increase when employing more than two wavefront-sensing planes with the same field of view. A multiple field of view layer-oriented approach (Ragazzoni et al. 2001) has been proposed in order to partially overcome this limitation, employing two wavefrontsensing planes having a different FOV per plane. In addition, studies concerning the viability of reconstructing the signal to be applied to an additional DM without adding another detector are on-going (Farinato et al. 2001). Both techniques are beyond the scope of this study, which is devoted to comparing the optical and numerical layer-oriented measurements in terms of signal to noise ratio $[S N R]$.

\section{Computation of SNR}

In this section, analytical formulae for the numerical and optical layer oriented measurements are developed. A large number of variables are involved and they are listed in Table 1 . We assume that the sampling is adjusted to the local atmospheric conditions in each layer for the OPTLO, that is: $d_{l}=r_{0, l}, \Delta t=\tau_{l}$, and to the global atmospheric parameters for the NUMLO: $d=r_{0}, \Delta_{t}=\tau$.

In order to fairly compare both techniques, we assume that the same type of wavefront sensor is used in both cases. For
OPTLO a pupil plane wavefront sensor is required. It could be for instance a pyramid wavefront sensor. The noise of such a sensor is comparable to that of a quad-cell Shack-Hartmann with a gain when operating in the closed-loop (Ragazzoni \& Farinato 1999; Esposito \& Ricardi 2001). To be conservative we will still consider in this paper the noise expressions related to a quad-cell Shack-Hartmann sensor (Rousset 1999):

signal photon noise $\sigma_{\mathrm{s}}^{2}=\pi^{2} \frac{1}{n_{\mathrm{ph}}}\left(\frac{\theta_{\mathrm{b}} d}{\lambda}\right)^{2} \operatorname{rad}^{2}$

detector noise $\sigma_{\mathrm{s}}^{2}=4 \pi^{2} \frac{R O N^{2}}{n_{\mathrm{ph}}^{2}}\left(\frac{\theta_{\mathrm{b}} d}{\lambda}\right)^{2} \operatorname{rad}^{2}$

background noise $\sigma_{\mathrm{s}}^{2}=\pi^{2} \frac{n_{\mathrm{bg}}}{n_{\mathrm{ph}}^{2}}\left(\frac{\theta_{\mathrm{b}} d}{\lambda}\right)^{2} \operatorname{rad}^{2}$

where $d$ is the subaperture diameter, $n_{\mathrm{ph}}$ is the number of photons per subaperture and exposure time, $R O N$ is the rms number of noise electrons per pixel and per frame, $n_{\text {bg }}$ is the total number of photoelectrons from the sky background distributed over the 4 pixels in the quadcell and $\theta_{\mathrm{b}}$ is the angular size of the subaperture image of the source. For the sake of simplicity we will first consider the case in which subaperture images are limited by diffraction, in which case $\theta_{\mathrm{b}}=\lambda / d$.

The ratio $1 / \sigma$, where $\sigma$ is the root square of the sum of the error variances due to photon, detector and background noise, can actually be interpreted as a signal to noise ratio in flux detection, as it gives the usual expression for the $S N R$ given by the ratio between the signal and square root of the sum of photon, detector and background noise:

$S N R=\frac{n_{\mathrm{ph}}}{\pi \sqrt{n_{\mathrm{ph}}+4 R O N^{2}+n_{\mathrm{bg}}}}$. 
Using the notation introduced in Table 1, and considering the optical layer-oriented measurements, we can write for measurements in the layer $l$ :

$$
\begin{aligned}
& S N R_{\mathrm{OPT}}^{l}(r)= \\
& \frac{\alpha_{l} d_{l}^{2} \tau_{l} Q_{\mathrm{e}} N_{\mathrm{ph}}^{l}(r)}{\pi \sqrt{\alpha_{l} d_{l}^{2} \tau_{l} Q_{\mathrm{e}} N_{\mathrm{ph}}^{l}(r)+4 R O N^{2}+\alpha_{l} d_{l}^{2} \tau_{l} Q_{\mathrm{e}} N_{\mathrm{bg}} N_{\mathrm{gs}}^{l}(r)}}
\end{aligned}
$$

where the signal term, as well as the photon noise and background noise terms are multiplied by $\alpha_{l}$, a factor which determines the fraction of light that is sent to detector $l$, with $\sum \alpha_{l}=1$. We consider a field of $1 \operatorname{arcsec}^{2}$ per guide star in the computation of the background photons, and this term is then multiplied by the number of stars that contribute to the corresponding subaperture.

NUMLO measurements are created from star-oriented measurements in which both spatial and temporal sampling are adjusted to match the global atmospheric characteristics. When reproducing the layer-oriented measurements, the same sampling as layer-oriented measures has to be used. If $d$ is the pixel size in the star-oriented measurements and $d_{l}$ is the pixel size in the layer-oriented measurements, the detector noise term has to be multiplied by a factor $\left(d_{l} / d\right)^{2}$ to account for the spatial binning required for layer-oriented spatial sampling and by a factor $\tau_{l} / \tau$ that is the summation of time samples to account for the increased exposure time in layer-oriented measurements. Also, a factor $N_{\mathrm{gs}}^{l}(r)$ accounts for the fact that star-oriented measurements employ one detector per guide star. Taking all this in consideration, we can write the following expression for the NUMLO SNR:

$$
\begin{aligned}
& S N R_{\mathrm{NUM}}^{l}(r)= \\
& \frac{d_{l}^{2} \tau_{l} Q_{\mathrm{e}} N_{\mathrm{ph}}^{l}(r)}{\pi \sqrt{d_{l}^{2} \tau_{l} Q_{\mathrm{e}} N_{\mathrm{ph}}^{l}(r)+4 R O N^{2} N_{\mathrm{gs}}^{l}(r) \frac{d_{l}^{2} \tau_{l}}{d^{2} \tau}+d_{l}^{2} \tau_{l} Q_{\mathrm{e}} N_{\mathrm{bg}} N_{\mathrm{gs}}^{l}(r)}}
\end{aligned}
$$

There is a potential gain in the OPTLO measurements where photons are co-added before detection, and thus, the resulting read-out noise is reduced as seen in the denominator of Eqs. (7) and (8). However, there is a penalization in terms of signal for this type of measurement as we divide the flux between the different wavefront-sensing planes. This penalization is represented by the factor $\alpha_{l}$ which accounts for the fraction of light that is sent to this wavefront-sensing plane, always smaller than one. These are opposite effects, hence the detailed study presented in this paper. Background noise behaves in the same way for both techniques except that the division of flux in the OPTLO measurements also affects background noise. Given:

$\gamma_{l}=\frac{d_{l}^{2}}{d^{2}} \frac{\tau_{l}}{\tau}$

we can write:

$$
\begin{aligned}
& S N R_{\mathrm{OPT}}^{l}(r)= \\
& \frac{\alpha_{l} \gamma_{l} d^{2} \tau Q_{\mathrm{e}} N_{\mathrm{ph}}^{l}(r)}{\pi \sqrt{\alpha_{l} \gamma_{l} d^{2} \tau Q_{\mathrm{e}} N_{\mathrm{ph}}^{l}(r)+4 R O N^{2}+\alpha_{l} \gamma_{l} d^{2} \tau Q_{\mathrm{e}} N_{\mathrm{bg}} N_{\mathrm{gs}}^{l}(r)}}
\end{aligned}
$$

$$
\begin{aligned}
& S N R_{\mathrm{NUM}}^{l}(r)= \\
& \frac{\gamma_{l} d^{2} \tau Q_{\mathrm{e}} N_{\mathrm{ph}}^{l}(r)}{\pi \sqrt{\gamma_{l} d^{2} \tau Q_{\mathrm{e}} N_{\mathrm{ph}}^{l}(r)+4 R O N^{2} N_{\mathrm{gs}}^{l}(r) \gamma_{l}+\gamma_{l} d^{2} \tau Q_{\mathrm{e}} N_{\mathrm{bg}} N_{\mathrm{gs}}^{l}(r)}} .
\end{aligned}
$$

In these expressions (Eqs. (10) and (11)), both the number of stars and the number of photons per $m^{2}$ per second depend on the position of the subaperture in the layer. For a wavefrontsensing plane conjugated to any layer but the telescope pupil, the footprints do not exactly overlap (see Fig. 2b), thus giving a density of photons and number of effective guide stars depending on the position of the subaperture in the detector. For a detector conjugated to the telescope pupil, all the star footprints overlap, and thus the number of guide stars and the density of photons is constant. In the following, we will make the simplifying hypothesis of:

$N_{\mathrm{gs}}^{l}(r)=N_{\mathrm{gs}}$, total number of guide stars,

$N_{\mathrm{ph}}^{l}(r)=N_{\mathrm{ph}}$, density of photons due to the contribution of all the guide stars.

As explained, this is perfectly valid in the pupil while in any other layer it means that we consider a portion of the layer where all the star footprints are super-imposed.

Equations (10) and (11) allow us to deduce the SNR ratio:

$$
\frac{S N R_{\mathrm{NUM}}^{l}}{S N R_{\mathrm{OPT}}^{l}}=\frac{1}{\alpha_{l}} \sqrt{\frac{\alpha_{l} \gamma_{l} r_{0}^{2} \tau Q_{\mathrm{e}}\left(N_{\mathrm{ph}}+N_{\mathrm{bg}} N_{\mathrm{gs}}\right)+4 R O N^{2}}{\gamma_{l} r_{0}^{2} \tau Q_{\mathrm{e}}\left(N_{\mathrm{ph}}+N_{\mathrm{bg}} N_{\mathrm{gs}}\right)+4 R O N^{2} N_{\mathrm{gs}} \gamma_{l}}} .
$$

In the simple case of having a detector without noise $(R O N=$ 0.), \frac{S N R_{\mathrm{NUM}}^{l}}{S N R_{\mathrm{OPT}}^{l}}=\frac{1}{\sqrt{\alpha_{l}}}>1$ as $0<\alpha_{l}<1$. The same result applies to very high flux cases where the read-out noise can be neglected. In this situation there will be no advantage for the OPTLO measurements. In any other situation, the $S N R$ ratio depends on a large number of factors: $N_{\mathrm{gs}}, N_{\mathrm{ph}}, N_{\mathrm{bg}}, R O N, r_{0}, \tau, \gamma_{l}$ and $\alpha_{l}$, as we will see in the following section.

Subaperture diffraction limited images occur when the subaperture diameter is smaller than the Fried diameter at the wavefront-sensing wavelength. This is the case studied so far. In order to generalize expressions 10 and 11 to account for the case of subaperture images limited by seeing, the same process is followed, departing from Eq. (3) and substituting the angular size of the image, $\theta_{\mathrm{b}}$, by $\lambda / r_{0}\left(\lambda_{\mathrm{wfs}}\right)$, corresponding to seeinglimited images (i.e. $r_{0}\left(\lambda_{\mathrm{wfs}}\right)<d$ ). This results in:

$$
\begin{aligned}
S N R_{\mathrm{OPT}}^{l}= & \frac{\alpha_{l} \gamma_{l} d^{2} \tau Q_{\mathrm{e}} N_{\mathrm{ph}}}{\pi \sqrt{\alpha_{l} \gamma_{l} d^{2} \tau Q_{\mathrm{e}} N_{\mathrm{ph}}+4 R O N^{2}+\alpha_{l} \gamma_{l} d^{2} \tau Q_{\mathrm{e}} N_{\mathrm{bg}} N_{\mathrm{gs}}}} \\
& \times \frac{r_{0}\left(\lambda_{\mathrm{wfs}}\right)}{d}
\end{aligned}
$$

$$
\begin{aligned}
S N R_{\mathrm{NUM}}^{l}= & \frac{\gamma_{l} d^{2} \tau Q_{\mathrm{e}} N_{\mathrm{ph}}}{\pi \sqrt{\gamma_{l} d^{2} \tau Q_{\mathrm{e}} N_{\mathrm{ph}}+4 R O N^{2} N_{\mathrm{gs}} \gamma_{l}+\gamma_{l} d^{2} \tau Q_{\mathrm{e}} N_{\mathrm{bg}} N_{\mathrm{gs}}}} \\
& \times \frac{r_{0}\left(\lambda_{\mathrm{wfs}}\right)}{d} .
\end{aligned}
$$

These are in fact the general expressions of the $S N R$ at the wavefront-sensing wavelength. When wavefront-sensing and 
imaging at the same wavelength, the subaperture size is set to match the size of the Fried parameter $\left(d=r_{0}\left(\lambda_{\mathrm{wfs}}\right)\right)$. In this case, the multiplying factor in Eqs. (13) and (14) is equal to one, and we find again the expressions (10) and (11).

The present article derives the $S N R$ expressions but of course the $S N R$ is related to the adaptive optics performance in terms of the Strehl Ratio (SR), generally approximated by the coherent energy $\exp \left(-\sigma_{\text {res }}^{2}\right)$. The residual phase variance $\sigma_{\text {res }}^{2}$ is a sum of different terms related to the temporal error, the fitting error and the wavefront-sensing noise. The contribution of the wavefront-sensing noise to the residual phase variance is derived from the subaperture noise variance propagated onto the correction modes and filtered by the servo-loop. At the imaging wavelength $\lambda_{\text {ima }}$, it is approximately given by:

$$
\begin{aligned}
\sigma_{\text {res, noise }}^{2} & =\alpha \frac{f_{\text {bw }}}{f_{\text {samp }}}\left(\frac{\lambda_{\mathrm{wfs}}}{\lambda_{\text {ima }}}\right)^{2} \sigma_{\text {subap, noise }}^{2} \\
& =\alpha \frac{f_{\text {bw }}}{f_{\text {samp }}}\left(\frac{\lambda_{\mathrm{wfs}}}{\lambda_{\text {ima }}}\right)^{2} \frac{1}{S N R^{2}} .
\end{aligned}
$$

Where $\alpha$ is the propagation coefficient on the corrected modes, $f_{\text {bw }}$ is the servo-loop bandwidth, $f_{\text {samp }}$ is the temporal sampling frequency, $S N R$ is the subaperture $S N R$ given in Eqs. (13) and (14). The loss in SR due to wavefront-sensing noise is therefore given by the multiplicative factor $\exp \left(-\sigma_{\text {res, noise }}^{2}\right)$, easily deduced from the wavefront-sensing $S N R$. For a high order system such as NAOS (Rousset et al. 2002), the factor $\alpha \frac{f_{\mathrm{bw}}}{f_{\mathrm{samp}}}$ is roughly 0.1 . When wavefront-sensing and imaging are performed at the same wavelength, the $S N R$ s of $1,0.5,0.3,0.2$ give respectively a loss in SR of 0.9, 0.7, 0.3 and 0.08 . The loss in performance is very rapid for $S N R$ s below 0.5 . Of course, in practice, when the $S N R$ is too small one reduces the wavefrontsensing exposure time to obtain a reasonable noise variance, at the expense of a larger temporal error.

\section{Discussion}

Analytical expressions have been obtained for the SNR corresponding to numerical and optical layer-oriented measurements, when the subaperture images are both in the diffraction and seeing limited regime. These expressions allow us to establish a comparison between the two techniques. It is seen that OPTLO measurements are favored when increasing the number of guide stars, and at low flux regimes.

OPTLO measurements are penalized by the increase of the number of measuring planes due to light splitting. On the contrary, the increase of the number of guide stars is a clear advantage of this approach, leading to significantly improved performance.

The analytical expressions given in Sect. 3 can be applied to any particular case, but it is useful and illustrative to see in a few figures the tendency of OPTLO and NUMLO approaches for some representative systems in adaptive optics. These graphics together with an extensive discussion can be found in the Appendix.

Acknowledgements. The authors would like to thank Dr. N. Devaney for his careful revision of this paper, and Thierry Fusco, Markus Kasper and Stefan Hippler for useful discussions. This work has been funded by the European Research and Training Network Adaptive optics for Extremely Large Telescopes with Contract HRPN-CT-2000-00147.

\section{References}

Babcock, H. W. 1953, PASP, 65, 229

Beckers, J. M. 1989, in Proc. SPIE Active Telescope Systems, ed. F. J. Roddier, 1114, 215-0

Bessell, M. S. 1979, PASP, 91, 589

Dicke, R. H. 1975, ApJ, 198, 605

Diolaiti, E., Ragazzoni, R., \& Tordi, M. 2001, A\&A, 372, 710

Esposito, S., \& Ricardi, A. 2001, A\&A, 369, L9

Farinato, J., Fedrigo, E., Marchetti, E., \& Ragazzoni, R. 2001, in Beyond Conventional Adaptive Optics, Venice, ESO Conf. \& Workshop Proc., 58

Flicker, R., Rigaut, F., \& Ellerbroek, B. 2000, in Adaptive Optical Systems Technology, ed. P. Wizinowich, Proc. SPIE, 4007, 1032

Graves, J. E., Northcott, M. J., Roddier, F. J., et al. 2000, in Adaptive Optical Systems Technology, ed. P. L. Wizinowich, Proc. SPIE, 4007, 26

Le Louarn, M., Hubin, N., Sarazin, M., \& Tokovinin, A. 2000, MNRAS, 317, 535

Ragazzoni, R. 1996, J. Mod. Opt., 43

Ragazzoni, R. 2000, in Proceedings of the Backaskog workshop on extremely large telescopes, 175

Ragazzoni, R., Diolaiti, E., Farinato, J., et al. 2001, in Beyond Conventional Adaptive Optics, ESO Conf. \& Workshop Proc., 58

Ragazzoni, R., \& Farinato, J. 1999, A\&A, 350, L23

Ragazzoni, R., Farinato, J., \& Marchetti, E. 2000, in Adaptive Optical Systems Technology, ed. P. L. Wizinowich, Proc. SPIE, 4007, 1076

Ragazzoni, R., Herbst, T., Andersen, D., et al. 2002, in Adaptive Optical Systems Technologies II, Proc. SPIE, 4839, 536

Rousset, G. 1999, Adaptive optics in astronomy (Cambridge University Press)

Rousset, G., Fontanella, J.-C., Kern, P., et al. 1990, A\&A, 230, 29

Rousset, G., Lacombe, F., Puget, P., et al. 2000, in Adaptive Optical Systems Technology, ed. P. L. Wizinowich, Proc. SPIE, 72, 4007

Rousset, G., Lacombe, F., Puget, P., et al. 2002, in Adaptive Optical System Technology II, Proc. SPIE (Bellingham, Washington: SPIE), 4839

Tallon, M., \& Foy, R. 1990, A\&A, 235, 549

Wilson, W. 1972, AJ, 177, 533

Wizinowich, P. L., Acton, D. S., Lai, O., et al. 2000, in Adaptive Optical Systems Technology, ed. P. L. Wizinowich, Proc. SPIE, 4007, 2 
D. Bello et al.: Signal to noise ratio of layer-oriented measurements, Online Material p 1

\section{Online Material}




\section{Appendix A: Study cases}

We have chosen three scenarios that are representative of different adaptive optics system characteristics:

- Case 1: wavefront-sensing and imaging in $K$ band $(2.20 \mu \mathrm{m})$.

- Case 2: wavefront-sensing and imaging in $R$ band $(0.64 \mu \mathrm{m})$.

- Case 3: wavefront-sensing in $R$ band and imaging in $K$ band.

Before studying these cases in the MCAO context, let us briefly recall the correspondences with current classical adaptive optics systems. Case 3 corresponds to most astronomical adaptive optics systems in service. In such conditions the wavefrontsensing $R O N$ is small but the wavefront-sensing channel is seeing limited. Case 1 is interesting to extend the sky coverage to regions where no bright visible sources are available. The first astronomical system to implement this option was NAOS on the VTL (Rousset et al. 2002). The RON is larger, wavefrontsensing is however diffraction limited. Case 2 corresponds to a system providing a good correction in the visible. This is a new perspective for adaptive optics systems and has never yet been implemented due to technological limitations. It would correspond to a small $R O N$ and to diffraction limited wavefrontsensing.

Since the $S N R$ depends on a large number of parameters, we decided to select the value of some of them, given in Table A.1. This table shows the wavefront-sensing and correction wavelength corresponding to each case. The bandwidth is equal for the three cases, $\Delta \lambda=0.4 \mu \mathrm{m}$. As a first approximation, the quantum efficiency $\left(Q_{\mathrm{e}}\right)$ is also equal for the three cases, even if it could be smaller for infrared detectors. We recall that in this work, the $Q_{\mathrm{e}}$ accounts also for optical transmission, as stated in Table 1. The Fried parameter corresponds to a seeing of 0.7 arcsec in the visible. The $R O N$ values correspond to the state-of-art of visible and infrared (Rousset et al. 2002) detectors accounting for the fast read-out frequency required by wavefront-sensing. The term $m_{\mathrm{bg}}$ accounts for the sky background magnitude at the wavefront-sensing wavelength. The integration time is taken to be constant and equal to $5.0 \mathrm{msec}$. Unless specified otherwise the number of guide stars is 10 .

In the following sections, we will consider two conjugate planes $\left(\alpha_{1}=\alpha_{2}=0.5\right)$ and study the dependence of OPTLO and NUMLO SNR on the amount of flux (Sect. A.1), we will investigate the gain in magnitude as a function of the number of guide stars (Sect. A.2), and the limit RON in order to have the same $S N R$ with both techniques (Sect. A.3). In Sect. A.4 we show the effect of increasing the number of DMs.

\section{A.1. SNR versus equivalent magnitude}

We represent the $S N R s$ as a function of the equivalent magnitude in the wavefront-sensing band. This equivalent magnitude is defined as the magnitude of a fictitious star with a number of photons per square meter per second equal to $N_{\mathrm{ph}}$, which is the total photon density given by the whole set of guide stars. The flux at wavelength $\lambda$ in units of $\mathrm{Wm}^{-2} \mu \mathrm{m}^{-1}$ is given by

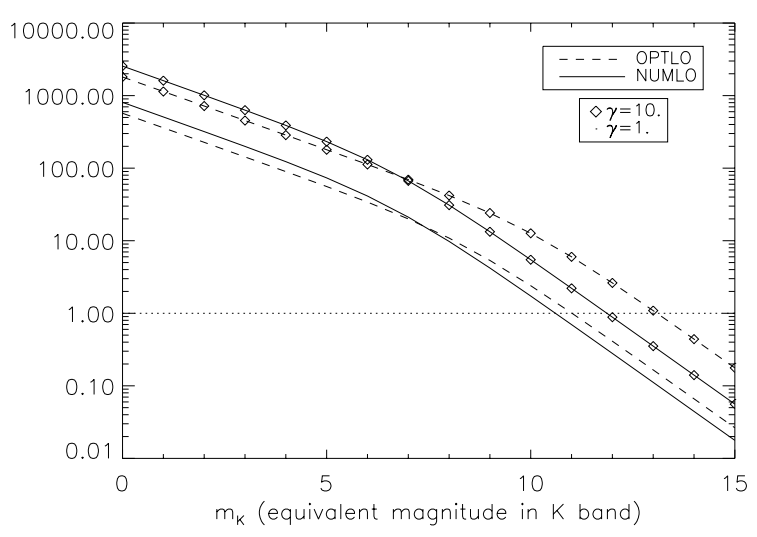

Fig. A.1. Numerical and optical $S N R$ when wavefront-sensing and imaging in the $K$ band (case 1 ) as a function of the equivalent magnitude in the $K$ band when employing 10 guide stars. Continuous lines correspond to numerical measurements and dashed lines correspond to optical measurements. Diamond symbols are used for $\gamma=10$., while no symbols are used for $\gamma=1$. Parameters are given in Table A.1.

$F_{0}(\lambda)=10^{-0.4 M_{v}-Z_{\mathrm{BAND}}}$, where $Z_{\mathrm{BAND}}$ is the zero point given in Table A.2 (Bessell 1979; Wilson 1972). The number of photons per square meter per second per $\mu \mathrm{m}$ is then found dividing the flux by the energy of the photon at the corresponding wavelength.

Figure A.1 shows the numerical and optical measurements $S N R$ as a function of the equivalent magnitude and for different values of $\gamma(1$ and 10) in the case of wavefront-sensing and correction in the $K$ band (case 1). $\gamma$ are chosen as a priori but reasonable values considering the turbulence profiles (Le Louarn et al. 2000). This figure demonstrates the main features of signal to noise ratio behavior. At small equivalent magnitude, the $S N R$ is dominated by the photon noise, and both detector and background noise are negligible. In this case, the NUMLO SNR is larger than the OPTLO by a factor $1 / \sqrt{\alpha_{l}}$ as explained in Sect. 3, due to the flux division between the conjugate planes done in the OPTLO configuration. The SNR is proportional to the square root of the number of photons in this region. Beyond equivalent magnitude $m_{k}=6$ OPTLO SNR becomes however larger. In this region, we are dominated by detector noise and the $S N R$ is proportional to the number of photons. For the values of $R O N$ considered (see Table A.1) which corresponds to state of the art infrared detectors, the detector noise term is larger than the contribution of background noise. Furthermore, when $\gamma$ is large the $R O N$ limitation for NUMLO is all the more apparent. In other words, the optical layer-oriented approach is particularly sensitive in high altitude layers where spatial and temporal sampling is optimized.

Notice that we have employed a constant exposure time. In a real system, when the $S N R$ is too low to allow the reconstruction of the phase, the exposure time is increased in order to increase the signal. In that case, the detector noise per frame will be the same, but the background noise will increase and become dominant. In that case the $S N R$ difference between the two types of measurements will decrease.

This curve allows us to understand the asymptotic behavior of the system (at very high and very low fluxes). The significant range of $S N R$ is of course more limited. As explained in 
Table A.1. Basic parameters used for the SNR estimation.

\begin{tabular}{cccccccccc}
\hline \hline & $\lambda_{\text {im }}(\mu \mathrm{m})$ & $\lambda_{\text {wfs }}(\mu \mathrm{m})$ & $\Delta \lambda(\mu \mathrm{m})$ & $Q_{\mathrm{e}}$ & $r_{0, \mathrm{wfs}}(\mathrm{m})$ & $r_{0, \mathrm{im}}(\mathrm{m})$ & $R O N$ & $m_{\mathrm{bg}}$ & $\tau(\mathrm{msec})$ \\
\hline Case 1 & 2.20 & 2.20 & 0.4 & 0.4 & 0.89 & 0.89 & 18.0 & 13.0 & 5.0 \\
Case 2 & 0.64 & 0.64 & 0.4 & 0.4 & 0.20 & 0.20 & 3.0 & 20.5 & 5.0 \\
Case 3 & 2.20 & 0.64 & 0.4 & 0.4 & 0.20 & 0.89 & 3.0 & 20.5 & 5.0 \\
\hline
\end{tabular}

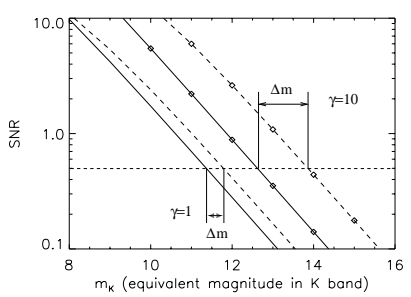

Case 1: wavefront-sensing, correction, $K$ band.

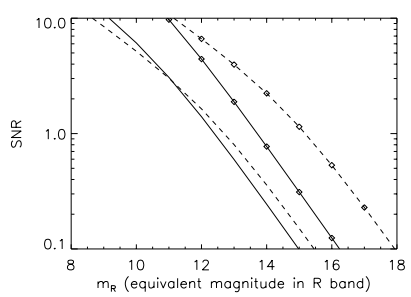

Case 2: wavefront-sensing, correction $R$ band.

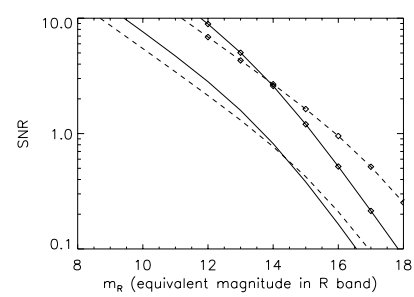

Case 3: wavefront sensing $R$ band, correction $K$ band.

Fig. A.2. $S N R$ versus equivalent magnitude at the wavefront-sensing wavelength. Continuous lines stand for numerical and dashed lines for optical. Curves corresponding to two values of $\gamma$ are shown: $\gamma=1$, without symbols; $\gamma=10$., diamond symbols. For the three cases we have assumed $N_{\mathrm{gs}}=10$.

Table A.2. Magnitude zero points employed in computing the equivalent magnitude corresponding to a number of photons per square meter and per second equal to $N_{\mathrm{ph}}$.

\begin{tabular}{ccc}
\hline \hline Band & $\lambda_{0}(\mu \mathrm{m})$ & $Z_{\text {BAND }}$ \\
\hline$R$ & 0.64 & 7.6408 \\
$K$ & 2.20 & 9.4367 \\
\hline
\end{tabular}

Sect. 3 for $S N R$ above 1 the impact on performance is very limited, and for $S N R$ below 0.2 the resulting SR is very small. A gain in $S N R$ in the range $0.2-1.0$ leads however to an important gain in performance. In case 3 , the same loss in performance is found for $S N R$ s multiplied by the wavelength ratio (imaging over wavefront-sensing wavelength 3.4 in our case). The pertinent range therefore becomes $0.68-3.4$.

Figures A.2a-c show the $S N R$ versus the equivalent magnitude in each of the wavefront-sensing bands, for the three cases defined in Sect. A. The SNR is limited to the range 0.1 to 10 and the number of guide stars is 10. Figure A.2a is in fact a zoom of the center of Fig. A.1. The case of $\gamma=1$ rather corresponds to the ground layer, where most of the turbulence is concentrated, and so the local Fried parameter is close to the global one. $\gamma=10$ could correspond to a high altitude layer with a local Fried parameter approximately three times larger than the global one, and an exposure time approximately 1.3 times larger than the global one. Increasing $\gamma$ induces an increase of the $S N R$ for both techniques as the signal itself is increased. However, in the NUMLO approach, this effect is balanced by the detector noise term which also increases with $\gamma$. Case 1 is the most favorable for OPTLO measurements (see Fig. A.2a). For both values of $\gamma$, the OPTLO SNR is larger than NUMLO $S N R$ in the domain shown. In this case, NUMLO measurements are penalized by the large $R O N$ of infrared detectors. In case 2, shown in Fig. A.2b, OPTLO measurements have larger SNR than NUMLO measurements for equivalent magnitudes larger than $m_{R}=11.5$ (if we consider that all the stars have the same magnitude, this corresponds to individual star magnitudes of $m_{R}=14.0$ ). Both in case 1 and 2, the wavefront-sensing wavelength is the same as the correction wavelength, and we have considered that subaperture images are diffraction limited. In case 3 , the wavefront-sensing is done in the $R$ band while the correction is done in the $K$ band, and thus subaperture images are seeing limited. This means a loss of $S N R$ by a factor $r_{0}\left(\lambda_{\mathrm{wfs}}\right) / r_{0}\left(\lambda_{\text {corr }}\right)$ with respect to the case when wavefrontsensing and correcting in the $K$ band, but the detector noise is less limiting here as we use detectors in the visible range with smaller RON. The background noise is also smaller when the wavefront-sensing is done in the visible with respect to the case when the wavefront-sensing is done in the infrared. The point at which NUMLO and OPTLO SNR crosses is now $m_{R} \approx 14$ (see Fig. A.2c). Star configurations with smaller equivalent magnitude will have better $S N R$ when doing NUMLO measurements, while with larger equivalent magnitude, the $S N R$ will be better for OPTLO measurements (as before, individual star magnitude would correspond to two and a half magnitudes more, that is $m_{R} \approx 16.5$ if stars are of the same magnitude).

\section{A.2. Magnitude gain}

Up to now, we have not considered the influence of the number of guide stars in the $S N R$ comparison. Figures A.3a-c show the gain in magnitude $\left(\Delta m=m_{\mathrm{OPTLO}}-m_{\mathrm{NUMLO}}\right)$ for the three study cases and for three different values of $S N R=0.5,1.0,2.0$ as a function of the number of guide stars. This gain in magnitude is computed as the difference in equivalent magnitude needed to achieve a fixed $S N R$ with the two techniques. An example of its definition is given in Fig. A.2a, where we show the magnitude gain for two values of $\gamma(1$ and 10) when the $S N R$ is equal to 0.5 . The numerical values corresponding to this example are $\Delta m \approx 1.5$ for $\gamma=10$ and $\Delta m \approx 0.5$ for $\gamma=1$. It has been defined so that a negative gain is favorable for the numerical approach while a positive gain is favorable for the optical ap- 


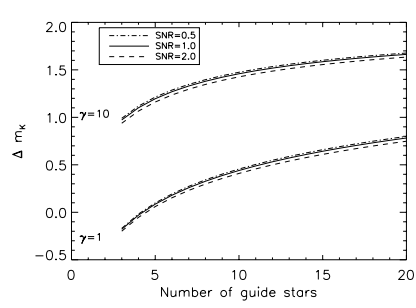

Case 1: wavefront-sensing, correction $K$ band.

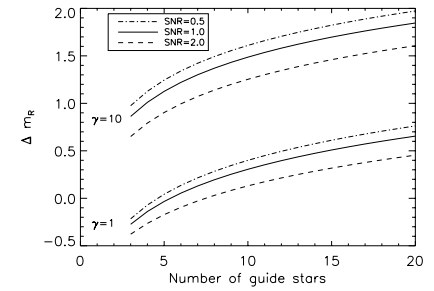

Case 2: wavefront-sensing, correction $R$ band.

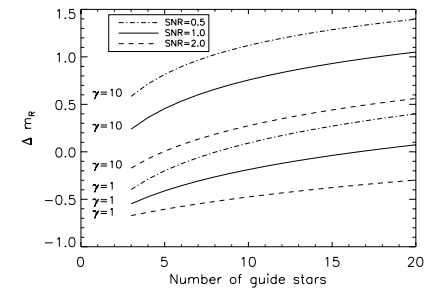

Case 3: wavefront-sensing $R$ band, correction $K$ band.

Fig. A.3. Gain in magnitude as a function of the number of guide stars, for $\gamma=1$. and $\gamma=10$. and several values of $S N R$. Dashed lines correspond to $S N R=2.0$, continuous lines to $S N R=1.0$ and dashed-dotted lines to $S N R=0.5$.

proach. The gain in magnitude depends strongly on the value of $\gamma$, that is to say, on the local atmospheric conditions of the layer with respect to the global atmospheric conditions as can be seen in Fig. A.3. Larger values of $\gamma$ mean an increase in terms of magnitude gain and increasing the number of guide stars also increases the magnitude gain.

Although the optical layer-oriented approach was proposed to employ and profit from a large number of guide stars, the number of guide stars that can be used is finally limited by the free space in the focal plane, and by the number of guide stars available in the FOV. Three to five guide stars will be reasonable for the north galactic pole while at the equator, the number of available guide stars could be about ten.

In the two first cases (Figs. A.3a and b) it can be seen that the OPTLO configuration can provide a magnitude gain even with a small number of guide stars (3-4), provided $\gamma>1$. In the third case, when the wavefront-sensing is done at the $R$ band and correction at the $K$ band, Fig. A.2c shows that the point where the NUMLO and OPTLO SNR curves cross is displaced towards larger magnitudes, thus for the chosen values of $S N R=0.5,1.0,2.0$, the gain is more modest than in the other two cases, as can be seen in Fig. A.3c.

The magnitude gain is relatively independent of $S N R$ values in case 1 , while in case 2 and 3 , for the same value of $\gamma$, the gain increases when the $S N R$ decreases. This is due to the fact that the magnitude gain depends on the point at which both OPTLO and NUMLO SNR curves cross and this point is determined by the weight of the detector noise term (always more limiting than the background noise term in this study) with respect to the signal. In case 3 for instance, the spatial sampling is relaxed by the fact that we are wavefront-sensing in the $R$ band to image in the $K$ band. In this case, the amount of flux per subaperture is larger and the detector noise is less limiting as explained also in Sect. A.1.

\section{A.3. Limiting $R O N$}

As shown in the previous sections, the potential gain of the OPTLO approach with respect to the NUMLO is based on a better management of the detector noise in the optical configuration. We have also seen that in the hypothetical case of $R O N=0$, numerical measurements will always be better than optical ones due to the penalty linked to dividing the flux between the different conjugate planes. Then one may ask for the situation of the limiting $R O N$, above which OPTLO SNR is larger than NUMLO $S N R$. This question is answered in Figs. A.4a-c where we show the limiting $R O N$ as a function of the equivalent magnitude in the three study cases and employing 10 guide stars. This value depends on the Fried parameter at the correction wavelength, the exposure time, number of background photons, number of guide stars, etc., although Figs. A.4a-c show only the dependence on equivalent magnitude and for two values of $\gamma(1$ and 10). We recall that detectors having a $R O N$ value larger than the one plotted in these figures will result in more efficient OPTLO measurements from the point of view of $S N R$, while detectors with $R O N$ smaller than that shown in the figures will result in more efficient NUMLO measurements.

There is a general behavior of the limiting $R O N$ in these figures; at high flux, the limiting $R O N$ is very high, while it decreases when the equivalent magnitude is increased and reaches an asymptotic value at faint flux $\left(R O N \approx 4 \mathrm{e}^{-} /\right.$pixel case 1 , $R O N \approx 0.15 \mathrm{e}^{-} /$pixel case $2, R O N \approx 0.6 \mathrm{e}^{-} /$pixel case 3$)$. In this region, due to the small value of $R O N$, background noise is dominant and this makes the limiting $R O N$ reach an asymptotic value.

In order to answer the question of the limiting $R O N$ we will place ourselves at moderately low $S N R$ because at large $S N R$, the difference between NUMLO and OPTLO SNR is not that important. We notice in Fig. A.4 that the limiting $R O N$ is different depending on the value of $\gamma$. In a real system, the staroriented measurements from which we construct the numerical layer-oriented measurements are done with wavefront sensors coupled to guide stars and with a single CCD for both layers. The SNR being always smaller when the $\gamma$ is smaller (that is, for the low altitude layers), this is the most limiting part of the system and we will consider the limiting RON given by the $S N R$ at $\gamma=1$. We can fix, for instance, $S N R=0.5$ in order to give an idea of the limiting $R O N$ for the three cases (although Fig. A.4 allows us to use any value of $S N R$ ). In order to achieve higher $S N R$ NUMLO that OPTLO with an $S N R=0.5$ for the measurements of the wavefront-sensing plane conjugate to the ground layer, we will need a $R O N$ lower than $0.6 \mathrm{e}^{-} /$pixel for case 2 and lower than $2.5 \mathrm{e}^{-} /$pixel for case 3 . Case 1 would need a RON lower than $4 \mathrm{e}^{-} /$pixel which is actually the asymptotic value.

The values required for infrared detector noise are still far from the state-of-the-art for high read-out frequencies, when 


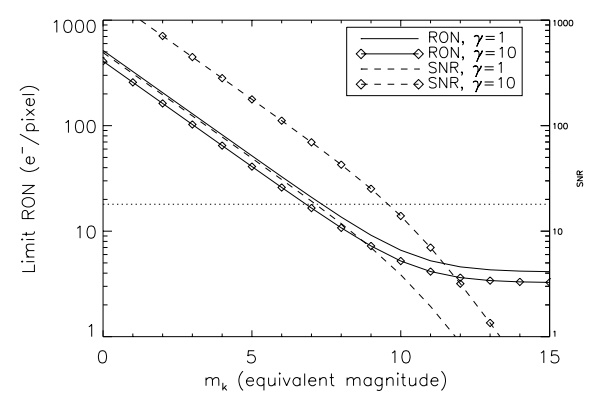

a) Case 1: sensing, imaging $K$ band.

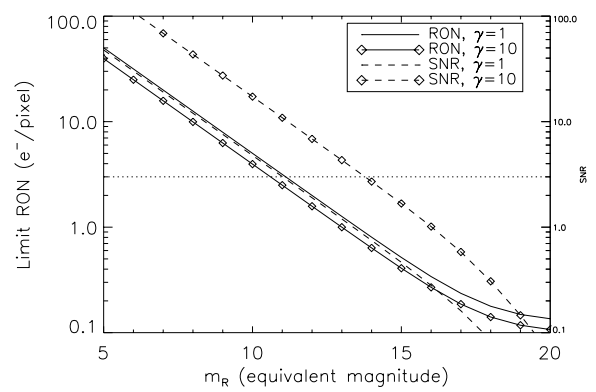

b) Case 2: sensing, imaging $R$ band

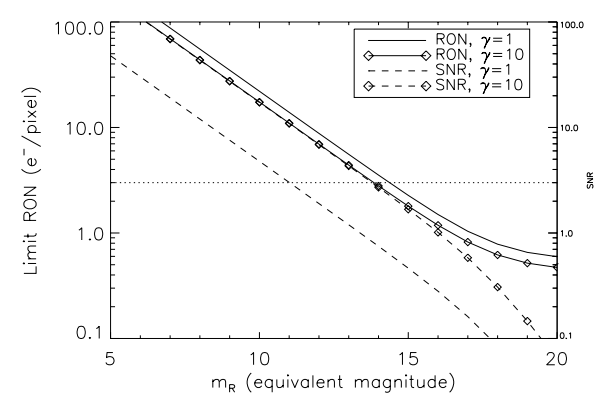

c) Case 3: sensing $R$ band, imaging $K$ band.

Fig. A.4. Limiting $R O N$ employing 10 guide stars, in order to have the same $S N R$ with NUMLO and OPTLO measurements. $R O N$ above this value will give better $S N R$ when using OPTLO measurements. Continuous lines correspond to RON while dashed lines correspond to SNR. No symbols are employed in the case of $\gamma=1$. while diamonds symbols are used for $\gamma=10$. Dotted lines have been drawn at $18 \mathrm{e}^{-} / \mathrm{pixel}$ in case 1 , and $3 \mathrm{e}^{-} /$pixel in cases 2 and 3, corresponding to the state-of-art of infrared and visible detectors respectively.

both the wavefront-sensing and correction are done in the $K$ band (case 1). In the case of wavefront-sensing in the $R$ band when imaging both in this band or in the $K$ band (case 2 and 3 ), the requirements in terms of detector noise are not so far from state-of-the-art visible detector $R O N$ values especially when measuring in the visible and correcting in the infrared (case 3). Recently, Marconi Applied Technologies have presented a new CCD technology called E2V which holds the promise of achieving sub-electron noise. However, these devices will be affected by statistics corruption due to the fact that they give one count if one or more photons are detected, hence introducing an error each time that more than one photon is detected. The influence of the statistic corruption using a LLLCCD in the $S N R$ is however out of the scope of this work.

It should be noticed that the value of the limiting $R O N$ shown in Figs. A.4a-c corresponds to parameters given in Table A.1, and 10 stars. With a smaller number of stars (than would be the situation when the scientific target is not at the galactic plane), or under better global seeing conditions, the requirement in $R O N$ is relaxed and could match current detector specifications.

\section{A.4. Influence of the number of detectors in the optical layer-oriented approach}

Up to now we have considered a wavefront sensor with two wavefront-sensing planes conjugated to different altitudes, with the total flux equally shared between both $\left(\alpha_{1}=\alpha_{2}=0.5\right)$. Simulations show that two DMs could be sufficient for correcting a 2 arcmin FOV in a $8 \mathrm{~m}$ class telescope with a SR of about $50 \%$, but higher order correction will need a larger number of DMs. The MCAO system planned for the Gemini telescope will employ three DMs and that will be also the case for the Large Binocular Telescopes (Flicker et al. 2000; Ragazzoni et al. 2002). In the case of future extremely large telescopes it is still not clear if a larger number of DMs will be necessary to ensure atmospheric turbulence correction. In that case, in the OPTLO configuration the straightforward solution (although not the only one, see for instance Ragazzoni et al. 2001; Farinato et al. 2001) will be to employ a wavefront sensor with more than two detectors. We will analyze in this Subsection the effect of increasing the number of deformable mirrors and therefore the number of detectors in terms of SNR. We will consider for OPTLO that light is split equally between all the detectors. We recall that in the numerical approach there is no loss in terms of SNR associated with increasing the number of deformable mirrors.

Figure A.5 shows the gain in magnitude as a function of SNR for four values of $\alpha=1.0,0.5,0.33$ and 0.25 corresponding to having 1 to 4 deformable mirrors, and two values of $\gamma=1$ and 10. The number of stars is 5 and 10 (Figs. A.5a and $\mathrm{b}$ respectively), and the wavefront-sensing and correction is done at the $R$ band. At high flux, the gain in magnitude is given by $\Delta m_{R}=2.5 \log _{10}(\alpha)$. It is negative when $\alpha$ is smaller 


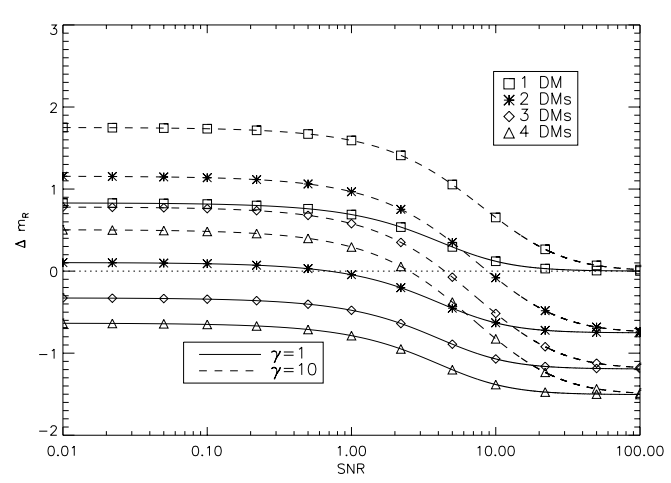

a) Five guide stars.

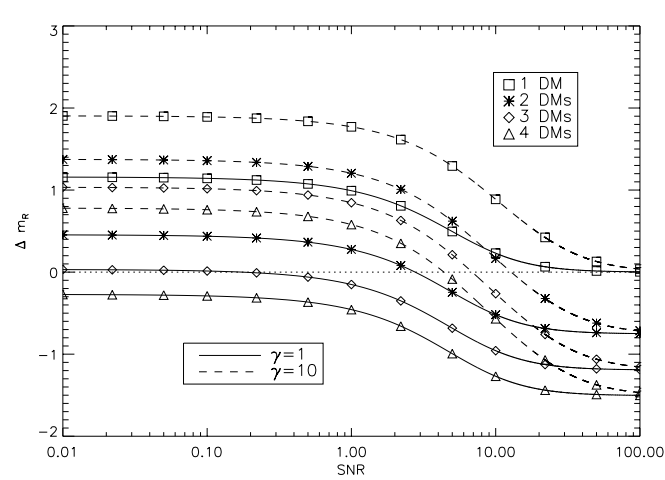

b) Ten guide stars.

Fig. A.5. Gain in magnitude as a function of $S N R$ for several values of $\alpha$ and $\gamma$ when wavefront-sensing and imaging in the $R$ band (case 2). Continuous lines are used for $\gamma=1$ and dashed lines for $\gamma=10$. Symbols are also used for the different numbers of DMs (i.e. different values of $\alpha$ ): squares for $1 \mathrm{DM}$, asterisks for $2 \mathrm{DMs}$, diamonds for $3 \mathrm{DMs}$ and triangles for $4 \mathrm{DMs}$.

than unity: when employing more than one detector the gain at high flux is actually in favor of the NUMLO configuration. At faint flux, in this case, read-out noise dominates and the gain in magnitude is $\Delta m_{R}=2.5 \log _{10}(\alpha)+1.25 \log _{10}\left(\gamma N_{\mathrm{gs}}\right)$. Comparison between Figs. A.5a and b emphasizes the fact that OPTLO measurements are favored when using a larger number of guide stars.

When wavefront-sensing and correcting in the $K$ band, the same type of curves are obtained, but the inflection point is displaced to higher $S N R$ ratios (about one order of magnitude). We are still limited by the $R O N$ as in the case of infrared detectors, $18 \mathrm{e}^{-} /$pixel for wavefront-sensing (Rousset et al. 2000).

In the case of wavefront-sensing in the $R$ band and imaging in the $K$ band, we will reach the regime of high flux very quickly ( $S N R \approx 10.0$ ), and from this value of $S N R$, the gain in magnitude is given by $\Delta m_{R}=2.5 \log _{10}(\alpha)$, which is negative (and thereby the gain will be for the NUMLO configuration).
In the ideal case of no detector noise, at very low flux, the gain will be $\Delta m=1.25 \log _{10}(\alpha)$, independent of the number of guide stars and the $\gamma$ factor, and the previous discussion is still valid.

Increasing the number of detectors in the OPTLO configuration can be done at the expense of some loss in the magnitude gain when operating at low flux and limited by detector noise. In this case, increasing the number of stars, or with a better $\gamma$, a positive gain can be maintained. When at low fluxes we are no longer limited by detector noise, but by background noise, the gain of the OPTLO configuration is lost, as the gain will be fixed by the value of $\alpha$ and independent of $\gamma$ and number of stars. 